\title{
Development and Validation of Ovarian Symptom Index-18 and Neurotoxicity-4 for Korean Patients with Ovarian, Fallopian Tube, or Primary Peritoneal Cancer
}

\author{
Maria Lee, $\mathrm{MD}, \mathrm{PhD}{ }^{1}$ \\ Yumi Lee, RN, $\mathrm{PhD}^{2}$ \\ Kidong Kim, MD, PhD 3 \\ Eun Young Park, MS ${ }^{4}$ \\ Myong Cheol Lim, MD, PhD 5,,6,7. \\ Jung-Sup Kim, $\mathrm{PhD}^{9}$ \\ Hee Seung Kim, MD, $P h D^{1}$ \\ Yong-Beom Kim, MD, PhD 3 \\ Yong-Man Kim, MD, PhD ${ }^{10}$ \\ Jungnam Joo, PhD ${ }^{4,7}$ \\ Sang Yoon Park, MD, PhD \\ Chel Hun Choi, MD, PhD ${ }^{11}$ \\ Jae-Hoon Kim, MD, PhD'12
}

*A list author's affiliations appears at the end of the paper.
Correspondence : Kidong Kim, MD, PhD Department of Obstetrics and Gynecology, Seoul National University Bundang Hospital, 82 Gumi-ro 173beon-gil, Bundang-gu, Seongnam 13620, Korea

Tel: 82-31-787-7262

Fax: 82-31-787-4054

E-mail: kidong.kim.md@gmail.com

Received August 1, 2017

Accepted March 5, 2018

Published Online March 7, 2018

*Maria Lee and Yumi Lee contributed equally to this study.

\section{Purpose}

The purpose of this study was to develop Korean versions of the National Comprehensive Cancer Network/Functional Assessment of Cancer Therapy (NCCN-FACT) Ovarian Symptom Index-18 (NFOSI-18) and FACT/Gynecologic Oncology Group (FACT-GOG) Neurotoxicity 4-item (NTX-4), evaluating their reliability and reproducibility.

\section{Materials and Methods}

In converting NFOSI-18 and NTX-4, the following steps were performed: forward translation, backward translation, expert review, pretest of preliminary format, and finalization of Korean versions (K-NFOSI-18 and K-NTX-4). Patients were enrolled from six institutions where each had completed chemotherapy for ovarian, tubal, or peritoneal cancer at least 1 month earlier. In addition to demographics obtained by questionnaire, all subjects were assessed via K-NFOSI-18, K-NTX-4, and a Korean version of the EuroQoL-5 Dimension. Internal structural validity and reliability were evaluated using item internal consistency, item discriminant validity, and Cronbach's $\alpha$. To evaluate test-retest reliability, K-NFOSI-18 and K-NTX-4 were readministered after 7-21 days, and intraclass correlation coefficients (ICCs) were calculated.

\section{Results}

Of the 250 women enrolled during the 3-month recruitment period, 13 withdrew or did not respond, leaving 237 (94.8\%) for the analyses. Mean patient age was $54.3 \pm 10.8$ years. Re-testing was performed in 190 patients (80.2\%). The total K-NFOSI-18 and K-NTX-4 scores were 49 (range, 20 to 72) and 9 (range, 0 to 16), respectively, with high reliability (Cronbach's $\alpha=0.84$ and 0.89 , respectively) and reproducibility (ICC $=0.77$ and 0.84 , respectively) achieved in retesting.

\section{Conclusion}

Both NFOSI-18 and NTX-4 were successfully developed in Korean with minimal modification. Each Korean version showed high internal consistency and reproducibility.

Key words

Ovarian neoplasms, Surveys, Questionnaires 


\section{Introduction}

In the United States alone, approximately 22,440 new cases of ovarian cancer and 14,080 related deaths were recorded for 2015 [1]. The incidence of ovarian cancer, which is now $1.5 \%$ annually in Korea [2], continues to climb. Ovarian cancer is typically diagnosed at an advanced stage of the disease (in more than $70 \%$ of patients). Relapse is common after firstline treatment, and prolonged therapeutic intervention is usually required $[3,4]$. Quality of life (QoL) is thus included with survival as an endpoint in clinical trials. However, there is scant evidence from the numerous studies conducted to date that palliative chemotherapeutic regimens administered for platinum-resistant ovarian cancer actually improve symptom control [5].

The National Comprehensive Cancer Network (NCCN)Functional Assessment of Cancer Therapy (FACT) Ovarian Symptom Index-18 (NFOSI-18), developed in the United States in 2011, helps assess symptoms that patients with advanced ovarian cancer may experience [6]. It is based on three subscales: disease-related symptoms, treatment-related symptoms, and general function/well-being Preliminary reliability indicators suggest good internal consistency, with a Cronbach's $\alpha$ of 0.80 . NFOSI-18 and its subscales have demonstrated significant positive associations with performance status. In a recent study validating the content of NFOSI-18, approximately $90 \%$ of participants rated each item as clear and understandable [7]. However, NFOSI-18 lacks a neurotoxicity subscale, and $18 \%$ of patients rendered spontaneous reports of neuropathy during open-ended questioning regarding symptoms. The FACT/Gynecology Oncology Group (GOG) Neurotoxicity 4-item (NTX-4) survey is a validated tool for assessing platinum/paclitaxel-induced neurologic symptoms [6,7].

The Korean Gynecology Oncology Group (KGOG) is considering participating in international GOG collaborative trials that use NFOSI-18 and NTX-4. As there are currently no validated Korean versions of these questionnaires, development and evaluation of Korean versions (K-NFOSI-18 and K-NTX-4) are required. Accordingly, the goals of this study were to adapt the existing NFOSI-18 and NTX-4 for Korean women and to evaluate the reliability and reproducibility of these adaptations.

\section{Materials and Methods}

\section{Study population}

This multicenter study, involving six institutions (Seoul National University Hospital, National Cancer Center, Seoul National University Bundang Hospital, Asan Medical Center, Samsung Medical Center, and Gangnam Severance Hospital), was conducted between August 2016 and October 2016. Participants were recruited by the respective Departments of Obstetrics and Gynecology and were consecutively enrolled. Inclusion criteria were as follows: (1) age 18 years or older; (2) diagnosis of ovarian, fallopian tube, or primary peritoneal cancer; (3) prior treatment with adjuvant chemotherapy or radiotherapy; and (4) ability to speak and understand the Korean language. Chemotherapeutic treatment within the 1 month prior to study enrolment, inability to understand the questionnaires, and refusal to participate were the criteria for exclusion.

The collected patient data included demographic variables (age, marital status, educational level, state of employment, family income, smoking/drinking habits, exercise activity, menopausal status, height, and weight) and disease characteristics (cancer stage, grade, duration of treatment, and recurrence status). To assess test-retest reliability, the questionnaires initially administered to patients were repeated in the same subjects 7-21 days later. All patients were asked to participate in this retesting. The Korean version of the EuroQoL-5 Dimension (K-EQ5D) questionnaire was also administered at the same time as the initial administration of K-NFOSI-18 and K-NTX-4 [8].

\section{NFOSI-18 and NTX-4 questionnaires}

Following baseline interviews, patients completed a Korean version of NFOSI-18 (K-NFOSI-18). NFOSI-18 consists of 18 items with four dimensions: disease-related symptoms, physical (nine items); disease-related symptoms, emotional (one item); treatment side effects (five items); and function/well-being (three items). Each item is scored from 0 ("not at all") to 4 ("very much") [6].

Following baseline interviews, patients also completed a Korean version of NTX-4 (K-NTX-4) [9]. NTX-4 consists of these four items: numbness or tingling in the feet, numbness or tingling in the hands, discomfort in the feet, and discomfort in the hands. Each item is scored from 0 ("not at all") to 4 ("very much").

K-NFOSI-18 and K-NTX-4 were developed through forward translation, backward translation, and discussion. Two independent researchers translated K-NFOSI-18 and K-NTX4 into Korean, and a bilingual (English and Korean) 
Table 1. Demographic and disease characteristics of study population

\begin{tabular}{|c|c|}
\hline Characteristic & No. $(\%)(n=237)$ \\
\hline Age, mean \pm SD (yr) & $54.27 \pm 10.75$ \\
\hline \multicolumn{2}{|l|}{ Marital status } \\
\hline Single & $26(11.0)$ \\
\hline Married & $191(80.6)$ \\
\hline Divorced & $9(3.8)$ \\
\hline Widowed & $11(4.6)$ \\
\hline \multicolumn{2}{|l|}{ Educational level (missing=2) } \\
\hline Elementary school or less & $17(7.3)$ \\
\hline Middle school & $20(8.5)$ \\
\hline High school & $72(30.6)$ \\
\hline College or above & $126(53.6)$ \\
\hline \multicolumn{2}{|l|}{ Employment (missing=1) } \\
\hline Employed & $67(28.4)$ \\
\hline Unemployed & 169 (71.6) \\
\hline \multicolumn{2}{|c|}{ Family income $(1,000$ won/mo) (missing=10) } \\
\hline$<200$ & $47(20.7)$ \\
\hline $200-300$ & $43(18.9)$ \\
\hline $300-400$ & $41(18.1)$ \\
\hline $400-500$ & $39(17.2)$ \\
\hline$>500$ & $57(25.1)$ \\
\hline \multicolumn{2}{|l|}{ Smoking } \\
\hline Current & $3(1.3)$ \\
\hline Past & $11(4.6)$ \\
\hline Nonsmoker & $223(94.1)$ \\
\hline \multicolumn{2}{|l|}{ Drinking (missing=1) } \\
\hline$>4$ times $/$ wk & 0 \\
\hline 2-3 times / wk & $3(1.3)$ \\
\hline Once/wk & $17(7.2)$ \\
\hline Nondrinker & $216(91.5)$ \\
\hline \multicolumn{2}{|l|}{ Regular exercise } \\
\hline Yes & $130(54.9)$ \\
\hline No & $107(45.1)$ \\
\hline \multicolumn{2}{|l|}{ Menopausal status } \\
\hline Yes & $216(91.1)$ \\
\hline No & $21(8.9)$ \\
\hline Height, mean \pm SD $(\mathrm{cm})$ & $157.88 \pm 6.17$ \\
\hline Weight, mean \pm SD $(\mathrm{kg})$ & $57.86 \pm 8.12$ \\
\hline $\begin{array}{l}\text { Treatment duration (missing }=6 \text { ), } \\
\text { median (min-max, mo) }\end{array}$ & $5(1-38)$ \\
\hline \multicolumn{2}{|l|}{ FIGO stage } \\
\hline 1 & $81(34.6)$ \\
\hline 2 & $30(12.8)$ \\
\hline 3 & $90(38.5)$ \\
\hline 4 & $27(11.5)$ \\
\hline Unknown & $6(2.6)$ \\
\hline
\end{tabular}

(Continued)
Table 1. Continued

$\begin{array}{lc}\text { Characteristic } & \text { No. }(\%)(\mathbf{n}=\mathbf{2 3 7}) \\ \text { Grade (missing=15) } & \\ 1 & 20(9.0) \\ 2 & 54(24.3) \\ 3 & 137(61.7) \\ 4 & 11(5.0) \\ \text { Chemotherapy regimens } & \\ \text { Paclitaxel+carboplatin } & 230(97.0) \\ \text { BEP } & 7(3.0) \\ \text { Recurrence or metastasis } & 61(25.7) \\ \text { Yes } & 176(74.3) \\ \text { No } & \end{array}$

$\mathrm{SD}$, standard deviation; min, minimum; max, maximum; FIGO, International Federation of Gynecology and Obstetrics; $\mathrm{BEP}$, bleomycin+etoposide+cisplatin.

researcher performed backward translation. The first Korean version was reviewed by a scholar of Korean literature. In September 2016, ten patients with ovarian cancer participated in a pilot test of both surveys at the Seoul National University Hospital. Based on the pilot results, item 13 ("I am bothered by side effects of treatment") was modified because the question was incomprehensible in the Korean language for two patients. Once revised, the finalized versions were administered to study participants at each hospital as interviewer-assisted self-report questionnaires.

\section{Data analysis}

Categorical variables were expressed as frequency (\%) and continuous variables as mean \pm standard deviation or median (range). To test the validity and reliability of questionnaires, item-level and dimension-level analyses were performed. In item-level evaluations, item internal consistency (IIC), and item discriminant validity (IDV) served as indices of internal structural validity. IIC reflects correlation between an item and all companion items in the same dimension, whereas IDV measures how well an item correlates with items in the same dimension, compared with items in other dimensions. In dimension-level evaluations, reliability was evaluated via Cronbach's $\alpha$, based on the total score or dimension score (in dimensions with more than two items). Reproducibility was evaluated through test-retest reliability and indicated by the intraclass correlation coefficient (ICC). The NFOSI-18 scoring guidelines ver. 2 (S1 Fig.) and NTX scoring guidelines ver. 4 (S2 Fig.) were used to calculate dimension scores. Responses were inverse-coded in K-NFOSI-18 and K-NTX-4, except for five questions in K-NFOSI-18 (C3, GF5, BMT5, GF3, and 
GF7), so that higher scores would represent better performance in both scores. Spearman's correlation coefficient was calculated to evaluate the external validity of K-NFOSI-18, K-NTX-4, and K-EQ5D scores. Differences in K-NFOSI-18 and K-NTX-4 scores according to recurrence status were compared using the Wilcoxon rank sum test to measure discriminant validity. All reported p-values were two-sided, with statistical significance set at $\mathrm{p}<0.05$. Statistical analyses were performed using $\mathrm{R}$ project freeware (ver. 3.3.2).

\section{Ethical statement}

The institutional review boards of each contributing institution (Seoul National University Hospital: IRB\#1607-206782, National Cancer Center: IRB\#2016-0194, Seoul National University Bundang Hospital: IRB\#1608-358-305, Asan Medical Center: IRB\#2016-0831, Samsung Medical Center: IRB\# 2016-07-087, and Gangnam Severance Hospital: IRB\#3-20160163) approved the study protocol, and all participants provided written informed consent.

\section{Results}

During the 3-month recruitment period, 250 women were enrolled in the study. The main analyses were limited to 237 patients $(94.8 \%)$, after excluding withdrawals and nonresponses. Re-testing without missing data was conducted in 190 patients (80.2\%). Table 1 displays socio-demographic and clinical characteristics of the study subjects. The mean age was $54.27 \pm 10.75$ years, with most patients registered as non-smokers, non-drinkers, and menopausal. More than half of the patients (55\%) exercised regularly. The median duration of treatment was 5 months. Paclitaxel-carboplatin based chemotherapy was received by $97 \%$ of patients. Cancer stages were distributed as follows: stage 1, 81/237 (34.6\%); stage 2, 30 / 237 (12.8\%); stage 3, 90 / 237 (38.5\%); and stage 4, $27 / 237$ (11.5\%). The overall rate of cancer relapse was $25.7 \%$.

Each item in K-NFOSI-18 and K-NTX-4 was determined according to the time of occurrence (i.e., within the previous 7 days). Median total scores for first-round survey administration were 49 (range, 20 to 72 ) and 9 (range, 0 to 16), respectively. The most frequent symptom was nausea (100\%), followed by cramping (99.6\%), vomiting (99.6\%), swelling of the stomach area (97.9\%), feeling ill (97.9\%), lack of energy (97.9\%), pain (97\%), constipation (96.6\%), and skin problems (95.7\%). Numbness or tingling sensations affected the hands $(91.9 \%)$ more commonly than the feet $(77.6 \%)$.

Table 2 provides a summary of structural validity, reliability, and reproducibility of K-NFOSI-18 and K-NTX-4.

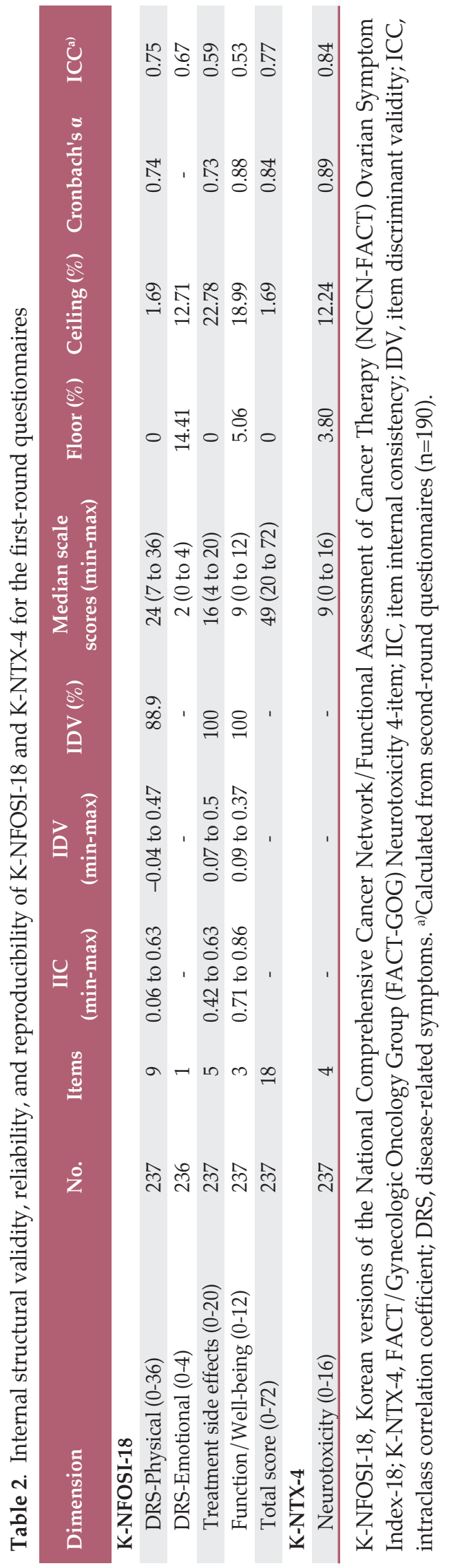

VOLUME 51 NUMBER 1 JANUARY 2019 
Table 3. Correlation between K-NFOSI-18 and K-NTX-4 scores

\begin{tabular}{lccccc} 
Dimension & NTX 1 & NTX 2 & NTX 3 & NTX 4 & K-NTX-4 total \\
\hline DRS-Physical (0-36) & 0.291 & 0.217 & 0.275 & 0.281 & 0.308 \\
DRS-Emotional (0-4) & 0.143 & 0.120 & 0.066 & 0.091 & 0.121 \\
Treatment side effects (0-20) & 0.340 & 0.266 & 0.314 & 0.222 & 0.330 \\
Function/Well-being (0-12) & 0.250 & 0.231 & 0.266 & 0.219 & 0.272 \\
Total NFOSI-18 score (0-72) & 0.331 & 0.274 & 0.317 & 0.281 & 0.348 \\
\hline
\end{tabular}

Data are Spearman's correlation coefficients for all dimension values. K-NFOSI-18, Korean versions of the National Comprehensive Cancer Network / Functional Assessment of Cancer Therapy (NCCN-FACT) Ovarian Symptom Index-18; K-NTX4, FACT/Gynecologic Oncology Group (FACT-GOG) Neurotoxicity 4-item; DRS, disease-related symptoms.

Table 4. Correlation between K-EQ5D items and VAS with K-NFOSI-18 scores

\begin{tabular}{lccrrrr} 
Dimension & $\begin{array}{c}\text { DRS- } \\
\text { Physical }\end{array}$ & $\begin{array}{c}\text { DRS- } \\
\text { Emotional }\end{array}$ & $\begin{array}{c}\text { Treatment } \\
\text { side effects }\end{array}$ & $\begin{array}{c}\text { Function/ } \\
\text { Well-being }\end{array}$ & $\begin{array}{c}\text { K-NFOSI-18 } \\
\text { total }\end{array}$ \\
Mobility & -0.292 & -0.102 & -0.209 & -0.320 & -0.324 \\
Self-care & -0.252 & -0.091 & -0.190 & -0.294 & -0.295 \\
Usual activities & -0.381 & -0.245 & -0.354 & -0.444 & -0.480 \\
Pain/Discomfort & -0.421 & -0.121 & -0.232 & -0.250 & -0.374 \\
Anxiety/Depression & -0.351 & -0.487 & -0.376 & -0.255 & -0.434 \\
VAS & 0.510 & 0.357 & 0.301 & 0.313 & 0.500 \\
\hline
\end{tabular}

Data are Spearman's correlation coefficients for all dimension values. K-EQ5D, Korean EuroQoL-5 Dimension survey; VAS, Visual Analogue Scale; K-NFOSI-18, Korean versions of the National Comprehensive Cancer Network/Functional Assessment of Cancer Therapy (NCCN-FACT) Ovarian Symptom Index-18; DRS, disease-related symptoms.

Table 5. Correlation between K-EQ5D items and VAS with K-NTX-4 scores

\begin{tabular}{|c|c|c|c|c|c|}
\hline Dimension & NTX 1 & NTX 2 & NTX 3 & NTX 4 & K-NTX-4 total \\
\hline Mobility & -0.399 & -0.383 & -0.352 & -0.475 & -0.471 \\
\hline Self-care & -0.237 & -0.205 & -0.277 & -0.263 & -0.287 \\
\hline Usual activities & -0.340 & -0.370 & -0.369 & -0.416 & -0.435 \\
\hline Pain/Discomfort & -0.423 & -0.371 & -0.409 & -0.460 & -0.482 \\
\hline Anxiety/Depression & -0.168 & -0.134 & -0.200 & -0.128 & -0.178 \\
\hline VAS & 0.314 & 0.363 & 0.317 & 0.422 & 0.411 \\
\hline
\end{tabular}

Data are Spearman's correlation coefficients for all dimension values. K-EQ5D, Korean EuroQoL-5 Dimension survey; VAS, Visual Analogue Scale; K-NTX-4, FACT / Gynecologic Oncology Group (FACT-GOG) Neurotoxicity 4-item.

K-NFOSI-18 exhibited high internal consistency and reliability (Cronbach's $\alpha=0.84$ ), with good reproducibility in re-testing: disease-related symptoms, physical (ICC $=0.75)$; diseaserelated symptoms, emotional (ICC $=0.67)$; treatment side effects (ICC $=0.59)$; function/well-being $(\mathrm{ICC}=0.53)$; and total score $(\mathrm{ICC}=0.77)$. K-NTX-4 also showed high reliability (Cronbach's $\alpha=0.89$ ) and reproducibility (ICC $=0.84$ ).
Table 3 lists the Spearman correlation coefficients for comparisons among K-NFOSI-18, K-NTX-4, and K-EQ5D scores for the first-round survey administration. There was weak positive correlation between total K-NFOSI-18 and K-NTX4 scores (Spearman correlation coefficient $=0.348$ ) (Table 3). K-EQ5D items showed weak-to-moderate correlation with K-NFOSI-18 and K-NTX-4 (Tables 4 and 5). K-EQ5D incor- 
porates five dimensions with three levels, generating one continuous score (Visual Analogue Scale). Correlation coefficients were negative because lower scores imply better status in K-EQ5D, whereas lower scores imply poorer status in K-NFOSI-18 and K-NTX-4 (Tables 4 and 5).

\section{Discussion}

In the present study, we developed reliable and reproducible Korean versions of standard NFOSI-18 and NTX-4 surveys (Cronbach's $\alpha=0.84$ and 0.89 , respectively; ICC $=0.77$ and 0.84 , respectively). Furthermore, there was positive correlation between total K-NFOSI-18 and K-NTX-4 scores.

A variety of validation tools are used to measure QoL in gynaecologic cancers, but they do not always align with the specific problems of patients with ovarian cancer $[10,11]$. Recently, ovarian cancer trials have added QoL as a secondary end-point [12,13], so tailoring questionnaires to specific countries for international use is assuming greater importance. The clinical impact of QoL is addressed in only $30 \%$ of such queries because of methodologic issues [14], underscoring the need to adopt Korean versions of the NFOSI-18 and NTX-4.

In this study, $>95 \%$ of patients exhibited disease-related symptoms in the physical realm; these were more common than emotional symptoms. In the dimension of treatment side effects, patients consistently experienced nausea (100\%) and vomiting (99.6\%). However, by adding neurologic symptoms to the body of questions, we found that most patients (87\%) experienced neurotoxicity as well, more often involving the hands than the feet. Although patients were excluded if they received chemotherapy within 1 month of study onset, it seems to contain a large number of patients currently on the chemotherapy course.

A distinct advantage of the present study is its large-scale, multicenter design. Furthermore, the short time required to complete the questionnaires ( $<10$ minutes) greatly encouraged compliance. Hence, the combined instrument (K-NFOSI-18 and K-NTX-4) may not be burdensome for patients. Although the ICC for constipation scoring in the K-NFOSI18 was $<0.80$, ICCs for most subscales and single items displayed good reproducibility. With ICC values of 0.7 or higher, two repeated test results may be expected to lie with a probability of $95 \%$. These findings indicate that the Korean version of NFOSI-18 is a stable adaptation. In discriminant validity analysis, a result greater than 0.85 , indicates that the two constructs overlap greatly and are likely measuring the same thing. In our study, IDV values were 88.9 to 100 , indicating that K-NFOSI-18 and K-NTX-4 have good discrimi- nant validity.

Previous studies have reported worse QoL in patients with disease recurrence [4,15-17]. However, other investigators have shown opposite results, generating controversy $[18,19]$. The present study confirmed (as expected) that patients with (vs. without) recurrence differed in terms of function/wellbeing scores. However, other dimensions, including diseaserelated symptoms and treatment side effects, showed no significant relationship with disease status.

Some study limitations are acknowledged. We did not assess changes in QoL over time, during chemotherapeutic cycles, or during multiple treatment cycles and courses. In addition, no patients hospitalized after surgery or chemotherapy were similarly surveyed to generate comparative results. Another limitation was the possibility that patients with symptoms may have been more likely to agree to participate in the study, leading to over-estimation of symptom rates. However, we chose the same inclusion criteria as those used in the study for which NFOSI-18 was first applied.

In conclusion, Korean versions of the NFOSI-18 and NTX4 questionnaires were successfully developed and demonstrated high internal consistency and reproducibility in the test population. Few modifications were required. Further efforts are required to address their reliability and validity in clinical research.

\section{Electronic Supplementary Material}

Supplementary materials are available at Cancer Research and Treatment website (https: // www.e-crt.org).

\section{Conflicts of Interest}

Conflict of interest relevant to this article was not reported.

\section{Author Details}

${ }^{1}$ Department of Obstetrics and Gynecology, Seoul National University College of Medicine, Seoul, 'Department of Nursing, Pukyong National University, Busan, ${ }^{3}$ Department of Obstetrics and Gynecology, Seoul National University Bundang Hospital, Seongnam, ${ }^{4}$ Biometrics Research Branch, Research Institute and Hospital, National Cancer Center, Goyang, ${ }^{5}$ Cancer Healthcare Research Branch, Center for Uterine Cancer, Research Institute and Hospital, National Cancer Center, Goyang, ${ }^{6}$ Center for Clinical Trials, Research Institute and Hospital, National Cancer Center, Goyang, ${ }^{7}$ Department of Cancer Control \& Population Health, Graduate School of Cancer Science and Policy, National Cancer Center, Goyang, Korea, ${ }^{8}$ Division of Gynecologic Oncology, Obstetrics and Gynecology, Irvine Medical Center, University of California, Orange, CA, USA, ${ }^{9}$ Korean Language and Literature, Kyung Hee University, Seoul, ${ }^{10}$ Department of Obstetrics and Gynecology, Asan 
Medical Center, University of Ulsan College of Medicine, Seoul, ${ }^{11}$ Department of Obstetrics and Gynecology, Samsung Medical Center, Sungkyunkwan University School of Medicine, Seoul, ${ }^{12}$ Depart- ment of Obstetrics and Gynecology, Gangnam Severance Hospital, Yonsei University College of Medicine, Seoul, Korea

\section{References}

1. Siegel RL, Miller KD, Jemal A. Cancer statistics, 2016. CA Cancer J Clin. 2016;66:7-30.

2. Lim MC, Moon EK, Shin A, Jung KW, Won YJ, Seo SS, et al. Incidence of cervical, endometrial, and ovarian cancer in Korea, 1999-2010. J Gynecol Oncol. 2013;24:298-302.

3. Cannistra SA. Cancer of the ovary. N Engl J Med. 2004;351: 2519-29.

4. Ezendam NP, Pijlman B, Bhugwandass C, Pruijt JF, Mols F, Vos MC, et al. Chemotherapy-induced peripheral neuropathy and its impact on health-related quality of life among ovarian cancer survivors: results from the population-based PROFILES registry. Gynecol Oncol. 2014;135:510-7.

5. Oronsky B, Ray CM, Spira AI, Trepel JB, Carter CA, Cottrill $\mathrm{HM}$. A brief review of the management of platinum-resistantplatinum-refractory ovarian cancer. Med Oncol. 2017;34:103.

6. Jensen SE, Rosenbloom SK, Beaumont JL, Abernethy A, Jacobsen $\mathrm{PB}$, Syrjala $\mathrm{K}$, et al. A new index of priority symptoms in advanced ovarian cancer. Gynecol Oncol. 2011;120:214-9.

7. Jensen SE, Kaiser K, Lacson L, Schink J, Cella D. Content validity of the NCCN-FACT ovarian symptom index-18 (NFOSI-18). Gynecol Oncol. 2015;136:317-22.

8. Kim MH, Cho YS, Uhm WS, Kim S, Bae SC. Cross-cultural adaptation and validation of the Korean version of the EQ-5D in patients with rheumatic diseases. Qual Life Res. 2005;14: 1401-6.

9. Calhoun EA, Welshman EE, Chang CH, Lurain JR, Fishman DA, Hunt TL, et al. Psychometric evaluation of the Functional Assessment of Cancer Therapy/Gynecologic Oncology Group-Neurotoxicity (Fact/GOG-Ntx) questionnaire for patients receiving systemic chemotherapy. Int J Gynecol Cancer. 2003;13:741-8.

10. Petersen MA, Groenvold M, Aaronson NK, Chie WC, Conroy $\mathrm{T}$, Costantini A, et al. Development of computerized adaptive testing (CAT) for the EORTC QLQ-C30 physical functioning dimension. Qual Life Res. 2011;20:479-90.

11. Basch E, Reeve BB, Mitchell SA, Clauser SB, Minasian LM, Dueck AC, et al. Development of the National Cancer Institute's patient-reported outcomes version of the common ter- minology criteria for adverse events (PRO-CTCAE). J Natl Cancer Inst. 2014;106:dju244.

12. Brundage M, Gropp M, Mefti F, Mann K, Lund B, Gebski V, et al. Health-related quality of life in recurrent platinum-sensitive ovarian cancer--results from the CALYPSO trial. Ann Oncol. 2012;23:2020-7.

13. Pignata S, Scambia G, Katsaros D, Gallo C, Pujade-Lauraine E, De Placido S, et al. Carboplatin plus paclitaxel once a week versus every 3 weeks in patients with advanced ovarian cancer (MITO-7): a randomised, multicentre, open-label, phase 3 trial. Lancet Oncol. 2014;15:396-405.

14. Efficace F, Jacobs M, Pusic A, Greimel E, Piciocchi A, Kieffer $\mathrm{JM}$, et al. Patient-reported outcomes in randomised controlled trials of gynaecological cancers: investigating methodological quality and impact on clinical decision-making. Eur J Cancer. 2014;50:1925-41.

15. Dunne S, Mooney O, Coffey L, Sharp L, Desmond D, Timon $\mathrm{C}$, et al. Psychological variables associated with quality of life following primary treatment for head and neck cancer: a systematic review of the literature from 2004 to 2015. Psychooncology. 2017;26:149-60.

16. Liavaag AH, Dorum A, Fossa SD, Trope C, Dahl AA. Controlled study of fatigue, quality of life, and somatic and mental morbidity in epithelial ovarian cancer survivors: how lucky are the lucky ones? J Clin Oncol. 2007;25:2049-56.

17. Monahan PO, Champion VL, Zhao Q, Miller AM, Gershenson D, Williams SD, et al. Case-control comparison of quality of life in long-term ovarian germ cell tumor survivors: a gynecologic oncology group study. J Psychosoc Oncol. 2008;26:19-42.

18. Mirabeau-Beale KL, Kornblith AB, Penson RT, Lee H, Goodman A, Campos SM, et al. Comparison of the quality of life of early and advanced stage ovarian cancer survivors. Gynecol Oncol. 2009;114:353-9.

19. Matei D, Miller AM, Monahan P, Gershenson D, Zhao Q, Cella $\mathrm{D}$, et al. Chronic physical effects and health care utilization in long-term ovarian germ cell tumor survivors: a Gynecologic Oncology Group study. J Clin Oncol. 2009;27:4142-9. 